\title{
Study on Wideband THz Backward Wave Oscillator Driven by Pseudospark-Sourced Sheet Electron Beam
}

\author{
Jin Zhang, Tianzhong Zhang, Yasir Alfadhl, Senior Member, IEEE, Xiaodong Chen, Fellow, IEEE, \\ Liang Zhang, Senior Member, IEEE, Huabi Yin and Adrian W. Cross, Member, IEEE
}

\begin{abstract}
A backward wave oscillator (BWO) based on a double staggered grating (DSG) slow wave structure (SWS) is investigated as a high-power wideband $\mathrm{THz}$ source, driven by a sheet electron beam emitting from a pseudospark plasma cathode. Firstly, the DSG SWS is optimized in simulation to have a suitable dispersion characteristic. Then, the BWO with a wideband output structure consisting of a tapered section of DSG and an L-shaped connector is modelled under the operating voltage of $24-38 \mathrm{kV}$ and the current density of $2-5 \times 10^{7} \mathrm{~A} / \mathrm{m}^{2}$ (beam current of $1.5-3.8 \mathrm{~A}$ ). A maximum power of $3.9 \mathrm{~kW}$ is obtained, and a wide bandwidth of over $38 \mathrm{GHz}(343-381 \mathrm{GHz})$ is achieved. The impact of fabricating errors of the SWS on the performance of the BWO is analyzed in simulation. The effects of the plasma in the interaction space on the BWO performance are also analyzed, showing that the plasma causes an increase in the oscillation frequency by $1.0 \%-1.2 \%$.
\end{abstract}

Index Terms-THz, backward wave oscillator (BWO), pseudospark cathode, sheet electron beam, wideband radiation.

\section{INTRODUCTION}

$\mathrm{O}$ VER recent years, considerable efforts have been put into developing $\mathrm{THz}$ sources for a variety of practical applications such as medical imaging [1], non-destructive imaging for quality control [2], security screening [3], communications with high data rate [4], plasma diagnostics [5], and electron paramagnetic resonance (EPR) system [6]. Driven by the demand of the market, there is a fast progress on $\mathrm{THz}$ sources, including the solid-state electronic devices, vacuum electron devices (VEDs), quantum cascade lasers (QCLs) and so on [7]. Solid-state electronic sources can deliver significant power at lower frequencies (below $100 \mathrm{GHz}$ ) but not at higher frequencies. On the other hand, QCLs are only capable of generating power in the frequency range of over $1 \mathrm{THz}$. In the frequency band of 0.1-1 THz, only VEDs, such as gyrotrons [8], free electron lasers (FELs) [9], backward wave oscillators (BWOs) [10], are capable of providing significant power.

Among the various VEDs, the BWO has the advantage of wide tunability of frequency [11]. The Russian corporation ISTOK have developed a series of cutting-edge BWO products, including some with power of $1-10 \mathrm{~mW}$ in the band 258-375 $\mathrm{GHz}$, and some high-frequency $(1.4 \mathrm{THz})$ products with power of up to $2 \mathrm{~mW}[12]$, using multirow interdigital or digit comb

The manuscript is submitted on $6^{\text {th }}$ May 2020.

This work was supported by the UK Engineering and Physical Sciences Research Council (EPSRC) under Grant EP/S009582/1.

This research utilised Queen Mary's Apocrita HPC facility, supported by QMUL Research-IT. http://doi.org/10.5281/zenodo.438045 slow wave structure (SWS) [13]. Several types of clinotrons (a variant of BWO) developed by Institute of Radio Physics and Electronics of the National Academy of Sciences (IRE NAS) in Ukraine demonstrate output power of $0.01-0.1 \mathrm{~W}$ in the band 272-334 GHz [14], using Lamellar grating SWS [15]. A combined effort by Stanford Linear Accelerator Center (SLAC), Beijing Vacuum Electronics Research Institute (BVERI) and Lancaster University is in progress to develop a double corrugated waveguide (DCW) BWO aiming at over $1 \mathrm{~W}$ continuous wave $(\mathrm{CW})$ at $0.346 \mathrm{THz}$ [16]. More recently, a novel folded waveguide (FWG) two-mode band-edge oscillator developed by BVERI delivers maximal output power of $1.51 \mathrm{~W}$ at $308.8 \mathrm{GHz}$ in BWO mode at a duty cycle of $65 \%$ [17]. Double staggered grating (DSG) SWS has also been used in the $\mathrm{THz}$ BWOs by Lancaster University [18] and the Institute of Electronics, Chinese Academy of Sciences [19]. Among these SWSs used in the BWOs, the DSG is competitive owing to several advantages. It can be relatively easily manufactured due to its 2-D planar structure[20], while fabrication of interdigital SWS encounters difficulties in the $\mathrm{THz}$ regime [21], and the THz DCW SWS must be manufactured using LIGA (German acronym for Lithography, Electroplating, and Molding) [16]. Also, the DSG SWS has relatively wider intrinsic frequency bandwidth, especially compared with the FWG. Besides, it has attractive features such as ease of assembly, mechanical robustness [19], robust thermal cooling capability, and capability to support a sheet electron beam [18]. Therefore, the DSG is an ideal SWS to be used in the THz BWOs.

The output power of the existing THz BWOs is relatively low due to a limited current density associated with thin electron beams. Also, the availability of THz BWOs is greatly restricted by high cost, large size due to magnetic focusing systems and high vacuum requirement. Therefore, we are proposing to tackle these challenges by developing a low-cost and compact high-power DSG THz BWO driven by a high-current psedudospark-sourced sheet electron beam.

The pseudospark plasma cathode has been developed in our groups to be used in VEDs to achieve high power THz radiation as compact and low-cost sources [22], due to its extraordinary features as follows. Firstly, the electron beam generated by pseudospark discharge is characterised by a very high current

J. Zhang, T. Zhang, Y. Alfadhl, and X. Chen are with the School of Electronic Engineering and Computer Science, Queen Marry University of London, London E1 4NS, U.K. (xiaodong.chen@qmul.ac.uk).

L. Zhang, H. Yin and A. W. Cross are with the Department of Physics, University of Strathclyde, Glasgow, G4 0NG, U.K. (a.w.cross@strath.ac.uk). 
density of the order of $10^{8} \mathrm{~A} / \mathrm{m}^{2}$ [23], which is much higher than that of conventional cathodes (usually $10^{6} \mathrm{~A} / \mathrm{m}^{2}[11]$ ). Secondly, the electron beam can form an ion channel while propagating through the thin gas chamber, leading to self-focus and eliminating the necessity of the magnetic focusing system [23], thus reducing the size. Thirdly, the formation of the electron beam is based on the repeated gas discharge in the hollow cathode, which will greatly extend the life of the cathode. Furthermore, the relatively lower vacuum requirement also simplifies the fabrication process.

In this paper, we have presented a wideband $\mathrm{THz} \mathrm{BWO}$ based on a DSG SWS driven by a psedudospark-sourced sheet electron beam. The wide bandwidth is achieved by introducing a unique output structure in the proposed BWO. Meanwhile, the sheet electron beam is used to further increase the beam current density, hence achieving high power.

\section{PSEUdOSPARK EleCtron SOURCE}

Fig. 1 shows a typical multi-gap pseudospark discharge setup consisting of a hollow cathode (HC) and an anode, which are separated by one or more insulators. The pseudospark discharge requires that the pressure in the $\mathrm{HC}$ is as low as $50-500 \mathrm{mTorr}$ so that the discharge is at the left-hand side of the Paschen curve [24]. When a high-voltage pulse is applied to the $\mathrm{HC}$, the pseudospark discharge will occur, and an electron beam with the current density of up to $10^{8} \mathrm{~A} / \mathrm{m}^{2}$ will be extracted through a hollow hole on the anode. As the beam travels, its front edge ionizes the background gas into plasma, and the following electrons expel part of the plasma electrons, forming an ionchannel, which confines the beam and eliminates the need for the magnetic focusing system [23]. By changing the parameters of the electric circuit powering the system, one can adjust the pulse duration of the electron beam, which is usually in the range of a few nanoseconds to a few hundred nanoseconds [23].

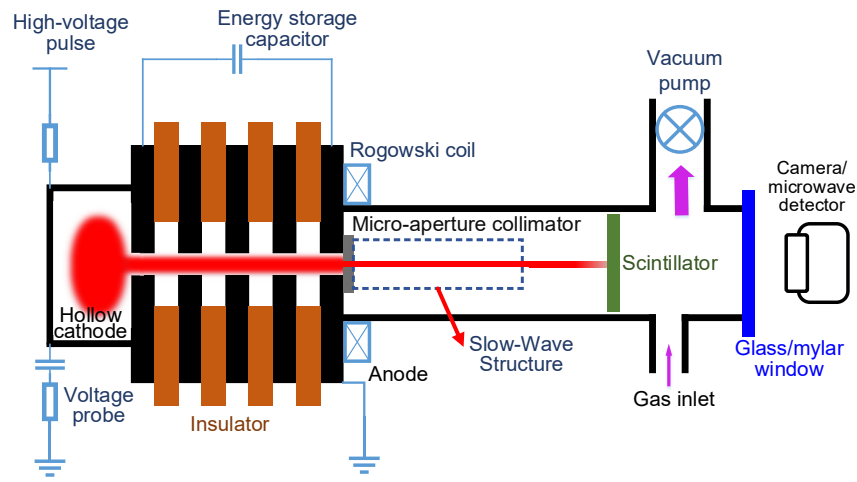

Fig. 1. Multi-gap pseudospark discharge setup.

A series of efforts have been put by our groups in developing VEDs driven by pseudospark-sourced electron beam to cover the millimetre-wave and $\mathrm{THz}$ bands, including a Ka-band Cherenkov maser [25], W-band and G-band extended interaction oscillators (EIOs) [26-27], and a G-band BWO [23].

\section{ANALYSIS ON DISPERSION CHARACTERISTICS}

The SWS is a core part of the BWO, supporting the transmission of a slow electromagnetic wave in synchronism with the electron beam. In our design, the DSG SWS as shown

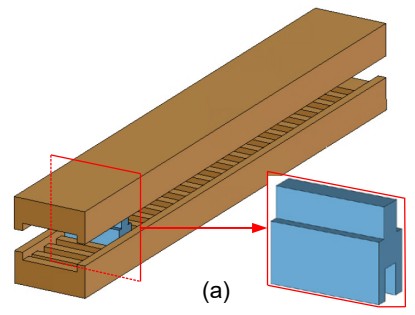



(b)

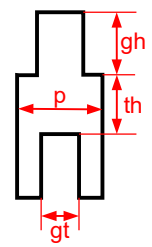

(c)
Fig. 2. DSG SWS in the BWO: (a) complete structure of DSG SWS; (b)-(c): cross-sectional/longitudinal view of a DSG unit with dimensional parameters.

TABLE I

DIMENSIONAL PARAMETERS OF DSG SWS

\begin{tabular}{lll}
\hline \hline Parameters & Value $/ \mathrm{mm}$ & \multicolumn{1}{c}{ Description } \\
\hline$w$ & 0.54 & Width \\
$t h$ & 0.14 & Height of beam tunnel \\
$p$ & 0.20 & Period \\
$g h$ & 0.15 & Height of grating \\
$g t$ & 0.09 & Thickness of grating \\
\hline \hline
\end{tabular}

in Fig. 2 is used [28]. Fig. 2 (a) shows the overall structure of the DSG SWS that consists of two rows of staggered gratings facing each other, and the space surrounded by metal in a unit is presented. Fig. 2(b) and Fig. 2(c) show the schematic of a DSG unit with the dimensional parameters labelled. The model of the DSG SWS is built and optimized in CST microwave studio. We use oxygen free copper (OFC) as the building material due to its high conductivity and good machinability, modelled by a lossy metal with a conductivity of $1.17 \times 10^{7} \mathrm{~S} / \mathrm{m}$ $\left(\sigma_{\mathrm{Cu}} / 5, \sigma_{\mathrm{Cu}}\right.$ is the $\mathrm{OFC}$ conductivity at low frequencies), which is a reasonable estimate for the OFC conductivity in sub- $\mathrm{THz}$ range [29]. The obtained optimized dimensional parameters are shown in Table I. The dispersion characteristics of the DSG SWS are shown in Fig. 3. The intersection between the dispersion curves of mode 1 and the slow space-charge wave denotes the synchronism between them, where oscillation occurs. The dispersion of the slow space-charge wave for a $32-$ $\mathrm{kV}$ electron beam is given in Fig. 3 as an example, which intersects the backward wave harmonic of mode 1 at $379 \mathrm{GHz}$, meaning the predicted working frequency is $379 \mathrm{GHz}$.

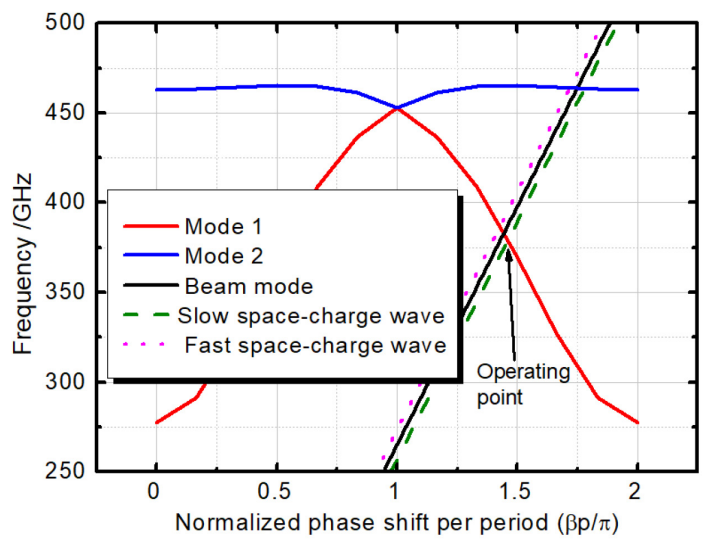

Fig. 3. Dispersion characteristics of modes 1 and 2 in the DSG SWS with beam modes of a 32-kV electron beam.

\section{Output Signal Simulation}

\section{A. Design of DSG BWO with a Wideband Output Structure}

Using the optimized DSG SWS in section III, we have designed the complete structure of the BWO, as shown in Fig. 


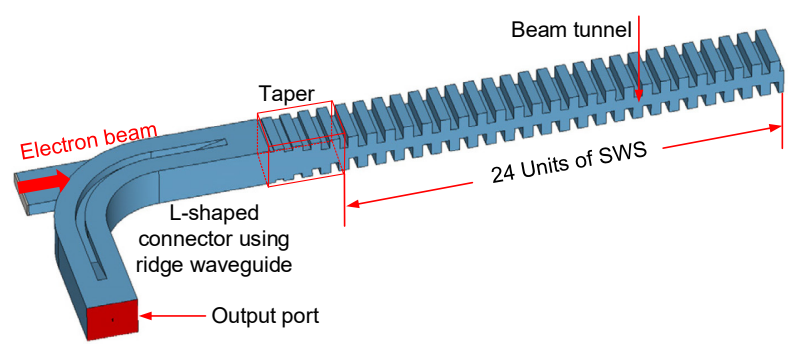

Fig. 4. Structure of the DSG BWO with a wideband output structure.

4. The emission area of the sheet electron beam has a rectangular shape $\left(0.54 \times 0.14 \mathrm{~mm}^{2}\right)$. The number of the units of SWS is optimized to 24 in simulation to obtain a stable and high-power signal. Fig. 4 also shows an output structure consisting of a tapered section of DSG and an L-shaped connector, which provides a smooth transition between the SWS and the output port, guiding the signal out with minimal reflection in the adjusting voltage range, so that the DSG BWO can work in a wide frequency band. The details of the wideband output structure are shown in Fig. 5.



(a)



(b)

Fig. 5. Wide-band output structure: (a) taper of DSG; (b) L-shaped connector using ridge waveguide.

The tapered section of DSG is shown in the pink dashed rectangle in Fig. 5(a), which provides a smooth transition between the normal DSG SWS and the L-shaped connector. The grating height decreases towards L-shaped connector in an arithmetic sequence as follows,

$$
\begin{aligned}
& g h_{0}=g h, \\
g h_{n}= & g h_{n-1}-d g,(n=1-9),
\end{aligned}
$$

where $g h_{n}(n=1-9)$ are the grating heights as shown in Fig. 5(a), $g h$ is the grating height of the normal DSG, $d g$ is the common difference (optimized to $0.011 \mathrm{~mm}$ ). Also, the grooves between the gratings are getting shallower as they approach the waveguide, which are equivalent to gradually increasing protrusion from the groove bottom in an arithmetic sequence,

$$
b h_{n}=n \cdot d b,(n=1-8),
$$

where $b h_{n}(\mathrm{n}=1-8)$ are the heights of the protrusion from the bottom in the grooves as shown in Fig. 5(a), $d b$ is the common difference, which is optimized as $0.005 \mathrm{~mm}$ in simulation.

The L-shaped connector is shown in Fig. 5(b), which is essentially a rectangular waveguide consisting of a straight part, a quarter-circle part, and another straight part. A pair of ridges are introduced on the top and bottom inner surfaces of the waveguide extending from the straight part of the waveguide through the quarter-circle part to the other straight part, as shown in the bottom subfigure of the Fig. 5(b). For each ridge, a pair of slopes are introduced on both ends to form a smooth transition. The cross-section of the ridge waveguide is shown in the top-left subfigure in Fig. 5(b), whose width and height are: $\mathrm{w}=0.54 \mathrm{~mm}$ (equal to the width of DSG SWS), $\mathrm{wh}=0.356 \mathrm{~mm}$, respectively; and the width and the height of the ridge are: $\mathrm{rw}=0.1 \mathrm{~mm}, \mathrm{rh}=0.09 \mathrm{~mm}$, respectively.

This output structure between the SWS and the output port can effectively reduce the reflection at the output port [30], which is crucial for a wideband operation. The simulation shows that without the output structure, the BWO would be working in several separate narrow bands.

\section{B. Analysis at a Single Frequency}

The particle-in-cell (PIC) simulation for the DSG BWO is conducted in CST particle studio. Fig. 6 shows the simulation results for the $32-\mathrm{kV}$ electron beam with a current density of $3 \times 10^{7} \mathrm{~A} / \mathrm{m}^{2}$ (beam current of $2.27 \mathrm{~A}$ ). The phase-space plot of the energy of the electrons is shown in Fig. 6(a), in which we can clearly observe the bunching effect during the process of the beam-wave interaction. Fig. 6(b) shows the port mode of the obtained signal at the output port, which is a $\mathrm{TE}_{10}$ mode. The time-domain and frequency-domain signals are shown in Fig. 6(c) and Fig. 6(d), respectively. Fig. 6(c) shows fast build-up of stable oscillation. Using the amplitude for calculation, we find that the output power is $1.8 \mathrm{~kW}$, the corresponding electronic efficiency being $2.5 \%$. Fig. $6(\mathrm{~d})$ shows that the signal frequency is $366.2 \mathrm{GHz}$, i.e., $3.4 \%$ lower than the predicted result (379 $\mathrm{GHz}$ ). This discrepancy is attributed to the downshift of the dispersion curve due to the presence of the electron beam [31].


Fig. 6. Simulation results of the DSG BWO. (a) Phase space plot of the energy of the electrons; (b) port mode at the output port; (c) time-domain output signal; (d) frequency-domain output signal. 

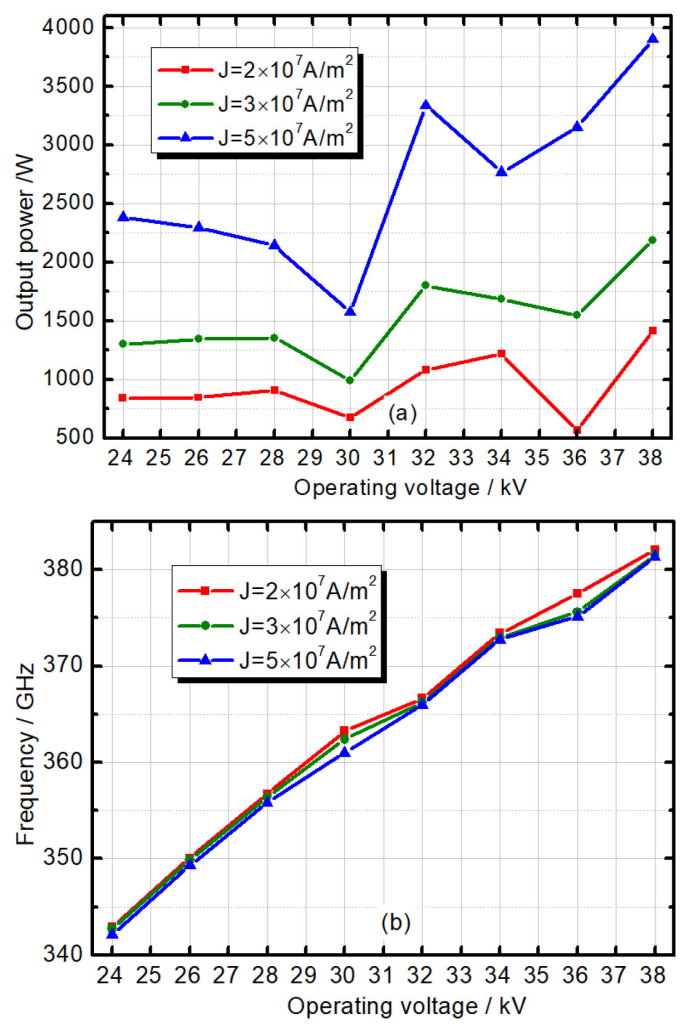

Fig. 7. Simulation results of DSG BWO in the voltage range of $24-38 \mathrm{kV}$ and the current density range of $2-5 \times 10^{7} \mathrm{~A} / \mathrm{m}^{2}$ (beam current of 1.5-3.8 A): (a) output power; (b) frequency.

\section{Study Over a Wide Band}

The DSG BWO over a range of voltage has been analyzed to examine its wideband operation. Also, it is necessary to study its performance over a range of current densities of the electron beam, as it could be lower than $10^{8} \mathrm{~A} / \mathrm{m}^{2}$. Therefore, we have adjusted the operating voltage from 24 to $38 \mathrm{kV}$, for current densities of $2-5 \times 10^{7} \mathrm{~A} / \mathrm{m}^{2}$ (beam current of 1.5-3.8 A) in the simulation, and obtained the results as shown in Fig. 7.

Fig. 7(a) shows the output power in the operating voltage range, which increases as the current density of the electron beam grows. The maximum power is $3.9 \mathrm{~kW}$ at $38 \mathrm{kV}$ when the beam current density is $5 \times 10^{7} \mathrm{~A} / \mathrm{m}^{2}$ (beam current of $3.8 \mathrm{~A}$ ), and the minimum power is $569 \mathrm{~W}$ at $36 \mathrm{kV}$ when the beam current density is $2 \times 10^{7} \mathrm{~A} / \mathrm{m}^{2}$. It shows that a high level of power is achieved in the voltage range of $24-38 \mathrm{kV}$ when the current density is above $2 \times 10^{7} \mathrm{~A} / \mathrm{m}^{2}$, which is the threshold current density in the DSG BWO. When the current density is below this threshold value, the BWO can only oscillate in a few discrete narrow bands. This indicates that the pseudosparksourced sheet electron beam is necessary for producing such a high current density. It is also observed that there are two output power dips at the operating voltage of $30 \mathrm{kV}$ and $36 \mathrm{kV}$, respectively. This is caused by a slight mismatch between the SWS and the output system. It is found that there was no power output around these two voltages when a simple output structure was adopted in the preliminary design of the BWO.

Fig. 7(b) shows the oscillation frequency of the DSG BWO in the operating voltage range for different current densities of the electron beam. The frequency increases as the operating voltage increases but decreases as the current density grows. The minimum frequency is less than $343 \mathrm{GHz}$ at $24 \mathrm{kV}$ while the maximum frequency is over $381 \mathrm{GHz}$ at $38 \mathrm{kV}$, showing that a bandwidth of over $38 \mathrm{GHz}$ is achieved. The increase of frequency with the growth of voltage can be explained with the Brillouin diagram in Fig. 3. As the voltage grows, the average velocity of the electrons also grows, meaning that the slope of the modes of the electron beam increases, and consequently the intersection point between the beam mode and the SWS mode is higher. The decrease of frequency with the growth of the beam current density can be explained as follows. When the current density in the electron beam is higher, the plasma frequency is also higher, meaning that the dispersion curve of the slow space-charge wave in Fig. 3 will shift downwards. Therefore, its intersection point with the dispersion curve of mode 1 will also shift downwards.

\section{ANALYSIS ON FABRICATING ERROR}

When manufacturing the parts of the DSG BWO, fabricating errors will occur, among which the errors of the grating height and the grating thickness influence the BWO performance more. Therefore, their influences are studied in simulation, for a beam with voltage of $32 \mathrm{kV}$ and current density of $3 \times 10^{7} \mathrm{~A} / \mathrm{m}^{2}$ (beam current of $2.27 \mathrm{~A}$ ). Firstly, the performance of the DSG BWO considering the error of the grating height is investigated. Considering the original height of $0.15 \mathrm{~mm}$, the simulation is conducted for values ranging from $0.11 \mathrm{~mm}$ to $0.19 \mathrm{~mm}$, obtaining the results as shown in Fig. 8. There is variation of power when the grating height changes, but the power remains over $1 \mathrm{~kW}$ in the entire range, showing that a significant level of power is maintained. Therefore, the robustness of the DSG BWO concerning the grating height is verified. The oscillation frequency decreases monotonically from $378 \mathrm{GHz}$ at $0.11 \mathrm{~mm}$ to $358 \mathrm{GHz}$ at $0.19 \mathrm{~mm}$ as the grating height increases.

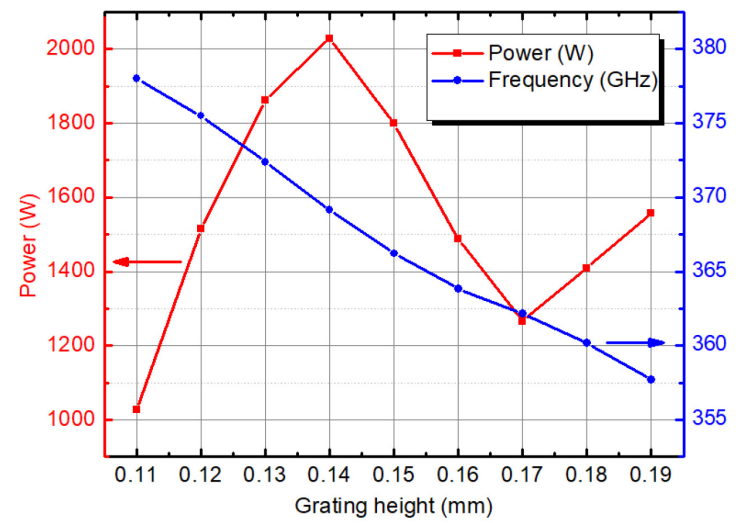

Fig. 8. Output power and frequency considering the error of the grating height.

Tolerance study on the grating thickness is conducted as well. The performance of the DSG BWO with grating thickness ranging from $0.06 \mathrm{~mm}$ to $0.12 \mathrm{~mm}$ is simulated, as shown in Fig. 9. When the grating thickness changes, the power ranges from $1.77 \mathrm{~kW}$ to $1.98 \mathrm{~kW}$, meaning a significant level of power is maintained. Therefore, the robustness of the DSG BWO concerning the grating thickness is verified. As the grating thickness increases from $0.06 \mathrm{~mm}$ to $0.12 \mathrm{~mm}$ and the oscillation frequency increases from $364 \mathrm{GHz}$ to over $371 \mathrm{GHz}$. 


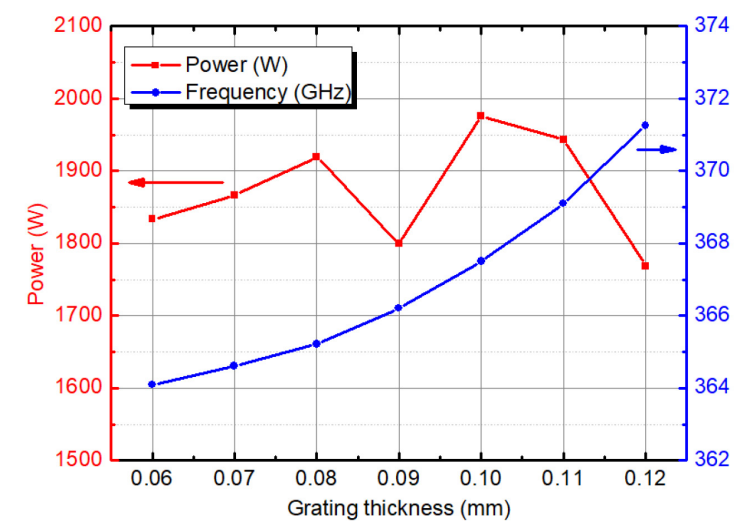

Fig. 9. Output power and frequency considering the error of grating thickness.

In summary, the results show that the DSG BWO is tolerant to the error of the grating height and thickness concerning power, but the oscillation frequency shifts. It is preferred to achieve a tolerance of $0.005 \mathrm{~mm}(5 \mu \mathrm{m})$ so that the conformity of the oscillation frequency in each unit of the SWS is guaranteed to avoid beat frequency. It is favourable to use the fabrication techniques such as LIGA and precision computer numerical control (CNC) machining.

\section{IMPACts of Plasma in Interaction Space}

When the DSG BWO is in operation, the sheet electron beam is extracted from the pseudospark cathode and travels in an ion channel in the beam tunnel, meaning that the interaction space is filled with low-pressure plasma, which could affect the performance of the DSG BWO.

The relative permittivity of the ideal vacuum is 1 , but it will change when the plasma is present. Using the Drude dispersion model, the ions in a plasma are assumed to be stationary while the electrons move freely, as the ion mass is much greater than the electron mass. Damping occurs due to elastic collisions between the moving electrons and the stationary ions, which can be described with the collision frequency $v_{c}$. Considering a specific plasma frequency $\omega_{p}$, the corresponding relative permittivity is given as,

$$
\varepsilon_{r}(\omega)=1-\frac{\omega_{p}^{2}}{\omega\left(\omega-i \nu_{c}\right)}
$$

where the plasma frequency is determined by,

$$
\omega_{p}=\sqrt{\frac{n_{e} e^{2}}{\varepsilon_{0} m_{e}}}
$$

and the collision frequency is given by the parameters of the gas in the pseudospark discharge, which in this case is Argon with a pressure of $60 \mathrm{~Pa}$ at room temperature. As shown in (4), the relative complex permittivity is a function of the frequency. The calculated real and imaginary part of the permittivity of the plasma is shown in Fig. 10. It shows that the plasma reduces the real part of the relative permittivity (0.94-0.97 in the band 300$400 \mathrm{GHz}$ ) compared to that in vacuum; the plasma increases the imaginary part of the relative permittivity $\left(2-6 \times 10^{-5}\right.$ in the band 300-400 GHz), indicating a slight attenuation. Fig. 10 also shows that the influence of the plasma is greater in the lowfrequency regime, but decreases in the high-frequency regime.

As CST particle studio does not allow PIC simulation for the DSG BWO with plasma, we simulate the dispersion

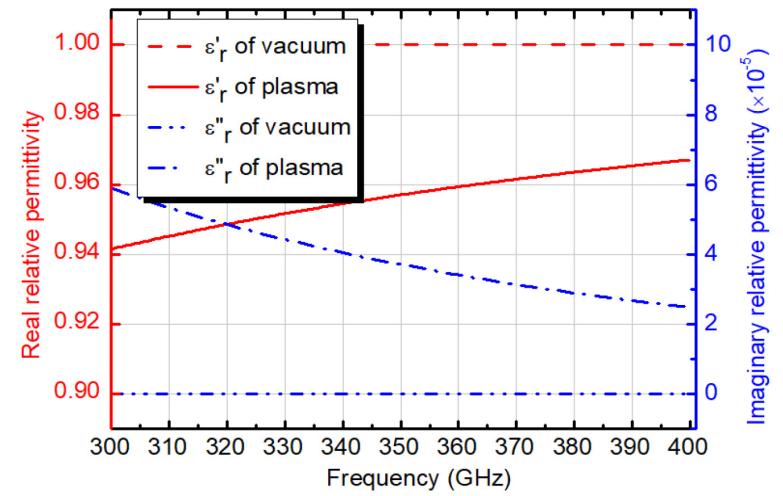

Fig. 10. Relative permittivity of plasma in the interaction space over the band 300-400 GHz.

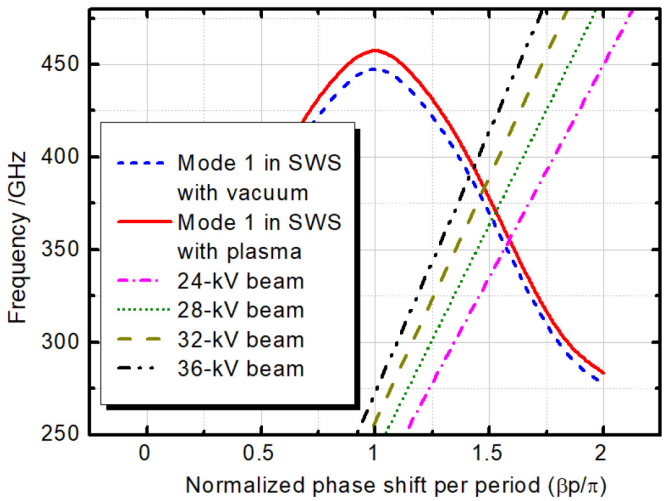

Fig. 11. Dispersion characteristics of the DSG SWS filled with plasma and beam modes at various voltages.

TABLE II

INCREASE OF OSCILLATION FREQUENCIES OF DSG BWO DUE TO PLASMA

\begin{tabular}{cllll}
\hline \hline $\begin{array}{c}\text { Beam } \\
\text { voltage } \\
/ \mathrm{kV}\end{array}$ & $\begin{array}{c}\text { Oscillation } \\
\text { frequency in } \\
\text { vacuum SWS } / \mathrm{GHz}\end{array}$ & $\begin{array}{c}\text { Oscillation } \\
\text { frequency in SWS } \\
\text { with plasma } / \mathrm{GHz}\end{array}$ & $\begin{array}{c}\text { Frequency } \\
\text { increase } \\
/ \mathrm{GHz}\end{array}$ & $\begin{array}{c}\text { Percentage } \\
\text { of increase }\end{array}$ \\
\hline 24 & 351.7 & 355.3 & 3.6 & $1.0 \%$ \\
28 & 366.8 & 370.7 & 3.9 & $1.1 \%$ \\
32 & 379.2 & 383.6 & 4.4 & $1.2 \%$ \\
36 & 389.9 & 394.6 & 4.7 & $1.2 \%$ \\
\hline \hline
\end{tabular}

characteristics of the DSG SWS filled with plasma in CST microwave studio, obtaining the results as shown in Fig. 11. It shows that the dispersion curve shifts upward a little, attributed to the reduction of the relative permittivity by plasma. Also, Fig. 11 includes the dispersion curves of the slow space-charge waves for electron beams with voltages from $24 \mathrm{kV}$ to $36 \mathrm{kV}$. It is noticeable that the intersection points between the beam modes and mode 1 in the SWS filled with plasma are higher than those in the vacuum SWS, meaning that the filled plasma causes an increase in the oscillation frequency. As shown in Table II, an increase of the oscillation frequency by 3.6-4.7 $\mathrm{GHz}(1.0 \%-1.2 \%)$ occurs due to the presence of the plasma.

\section{CONCLUSION}

In this paper, we have investigated a THz DSG BWO driven by a pseudospark-sourced sheet electron beam in simulation. The DSG SWS was optimized to exhibit a favourable dispersion characteristic. A wideband output structure consisting of a taper of DSG and an L-shaped connector was designed to provide a smooth transition between the SWS and the output port. 
Under the operating voltage of $24-38 \mathrm{kV}$ and current density of $2-5 \times 10^{7} \mathrm{~A} / \mathrm{m}^{2}$ (beam current of 1.5-3.8 A), a high level of output power up to $3.9 \mathrm{~kW}$ (at $38 \mathrm{kV}$ and $5 \times 10^{7} \mathrm{~A} / \mathrm{m}^{2}$ ) over a bandwidth of over $38 \mathrm{GHz}(343-381 \mathrm{GHz})$ is achieved in the proposed BWO. The effects of the fabrication errors of the height and thickness of the grating were assessed to show the robustness of this DSG BWO. And it is preferred to achieve a fabrication tolerance of no more than $5 \mu \mathrm{m}$ to guarantee the conformity of the oscillation frequency in different SWS units. The study on the effects of the plasma in the interaction space shows that the presence of plasma brings a reduction in the real part of the relative permittivity, and consequently the oscillation frequency increases by $1.0 \%-1.2 \%$.

\section{ACKNOWLEDGMENT}

Jin Zhang thanks Jie Xie of University of Electronic Science and Technology, China for his helpful advices.

\section{REFERENCES}

[1] Y. Liu, H. Liu, M. Tang, J. Huang, W. Liu, J. Dong, X. Chen, W. Fu, Y. Zhang, "The medical application of terahertz technology in non-invasive detection of cells and tissues: opportunities and challenges," RSC $A d v$., vol. 9, no. 17, pp. 9354-9363, Mar. 2019. DOI: 10.1039/C8RA10605C.

[2] I. Duling, D. Zimdars, "Terahertz imaging: revealing hidden defects," Nat. Photonics, vol. 3, no. 11, pp. 630-632, Nov. 2009. DOI: 10.1038/nphoton.2009.206.

[3] J. F. Federici, B. Schulkin, F. Huang, D. Gary, R. Barat, F. Oliveira, D. Zimdars, "THz imaging and sensing for security applications - explosives, weapons and drugs," Semicond Sci. Technol., vol. 20, no. 7, pp. S266S280, Jun. 2005. DOI: 10.1088/0268-1242/20/7/018.

[4] S. Koenig, D. Lopez-Diaz, J. Antes, F. Boes, R. Henneberger, A. Leuther, A. Tessmann, R. Schmogrow, D. Hillerkuss, R. Palmer, T. Zwick, "Wireless sub-THz communication system with high data rate," Nat. photonics, vol. 7, no. 12, pp. 977-981, Oct. 2013. DOI: 10.1038/NPHOTON.2013.275.

[5] B. H. Kolner, R. A. Buckles, P. M. Conklin, R. P. Scott, "Plasma characterization with terahertz pulses," IEEE J. Sel. Top Quantum Electron, vol. 14, no. 2, pp. 505-512, Apr. 2008. DOI: 10.1109/JSTQE.2007.913395.

[6] S. Takahashi, L. C. Brunel, D. T. Edwards, J. Van Tol, G. Ramian, S. Han, M. S. Sherwin, "Pulsed electron paramagnetic resonance spectroscopy powered by a free-electron laser," Nature, vol. 489, no. 7416, pp.409-413, Sep. 2012. DOI: $10.1038 /$ nature 11437.

[7] D. M. Mittleman, "Frontiers in terahertz sources and plasmonics," Nat. Photonics, vol. 7, no. 9, pp. 666-669, Sep. 2013. DOI: 10.1038/nphoton.2013.235.

[8] V. L. Bratman, Y. K. Kalynov, V. N. Manuilov, "Large-orbit gyrotron operation in the terahertz frequency range," Phys. Rev. Lett., vol. 102, no. 24, pp. 245101, Jun. 2009. DOI: 10.1103/PhysRevLett.102.245101.

[9] O. Shevchenko, V. Arbuzov, N. Vinokurov, P. Vobly, V. Volkov, Y. Getmanov, Y. Gorbachev, I. Davidyuk, O. Deychuly, E. Dementyev, B. Dovzhenko, "The Novosibirsk free electron laser-unique source of terahertz and infrared coherent radiation," Phys. Procedia, vol. 84, pp. 1318, Dec. 2016. DOI: 10.1016/j.phpro.2016.11.004.

[10] J. Feng, Y. Tang, D. Gamzina, X. Li, B. Popovic, M. Gonzalez, L. Himes, R. Barchfeld, H. Li, P. Pan, R. Letizia, "Fabrication of a 0.346-THz BWO for plasma diagnostics," IEEE Trans. Electron Devices, vol. 65, no. 6, pp. 2156-2163, Jun. 2018. DOI: 10.1109/TED.2018.2821683.

[11] J. H. Booske, R. J. Dobbs, C. D. Joye, C. L. Kory, G. R. Neil, G. S. Park, J. Park, R. J. Temkin, "Vacuum electronic high-power terahertz sources," IEEE Trans. Terahertz Sci. Technol., vol. 1, no. 1, pp. 54-75, Sept. 2011. DOI: 10.1109/TTHZ.2011.2151610.

[12] A. A. Borisov, U. A. Budzinsky, S. V. Bykovsky, A. V. Galdetskiy, A. N. Korolev, M. I. Lopin, A. A. Negirev, V. I. Pugnin, G. V. Ruvinsky, B. V. Sazonov, "The development of vacuum microwave devices in Istok," 2011 IEEE IVEC, Bangalore, India, 2011, pp. 437-438. DOI: 10.1109/IVEC.2011.5747063.

[13] A. Korolev, S. Zaitsev, I. Golenitskij, Y. Zhary, A. Zakurdayev, M. Lopin, P. Meleshkevich, E. Gelvich, A. Negirev, A. Pobedonostsev, V. Poognin, "Traditional and novel vacuum electron devices," IEEE Trans. Electron
Devices, vol.48, no.12, pp.2929-2937, Dec.2001. DOI: $10.1109 / 16.974731$.

[14] S. I. Molokovsky and A. D. Sushkov, "Methods of fields calculations," in Intense electron and ion beams, Berlin, Heidelberg, Germany: Springer Science \& Business Media, 2005, pp. 27-45.

[15] S. Ponomarenko, S. Kishko, V. Zavertanniy, E. Khutoryan, I. Lopatin, B. Yefimov, A. Kuleshov, "400-GHz continuous-wave clinotron oscillator," IEEE Trans. Plasma Sci., vol. 41, no. 1, pp. 82-86, Jan. 2013. DOI: 10.1109/TPS.2012.2226247.

[16] D. Gamzina, X. Li, C. Hurd, Y. Tang, X. Huang, Y. Zheng, L. Himes, M. Gonzalez, H. Li, P. Pan, R. Letizia, "Backward wave oscillator for high power generation at THz frequencies," in Proc. SPIE 10383, Terahertz Emitters, Receivers, and Applications VIII, San Diego, CA, USA, Aug. 2017, pp. 1038303. DOI: 10.1117/12.2273256.

[17] L. Zhang, J. Cai, X. Bian, X. Wu, J. Feng, "A novel THz forward and backward wave two-mode band-edge oscillator," IEEE Trans. Terahertz Sci. and Technol., to be published. DOI: 10.1109/TTHZ.2020.2972291.

[18] C. Paoloni, D. Gamzina, L. Himes, B. Popovic, R. Barchfeld, L. Yue, Y. Zheng, X. Tang, Y. Tang, P. Pan, H. Li, "THz backward-wave oscillators for plasma diagnostic in nuclear fusion," IEEE Trans. Plasma Sci., vol. 44, no. 4, pp. 369-376, Apr. 2016. DOI: 10.1109/TPS.2016.2541119.

[19] Q. Liu, Z. Wang, P. Liu, C. Du, H. Li, and A. Xu, "A THz backward-wave oscillator based on a double-grating rectangular waveguide," IEEE Trans. Electron Devices, vol. 60, no. 4, pp. 1463-1468, Apr. 2013. DOI: 10.1109/TED.2013.2246868.

[20] G. Shu, G. Zhang, W. He, "Design and measurement of a terahertz double staggered grating waveguide with an arc-shaped beam tunnel," IEEE Trans. Electron Devices, vol. 66, no. 11, pp. 4932-4937, Nov. 2019. DOI: 10.1109/TED.2019.2938551.

[21] M. Mineo and C. Paoloni, "Corrugated rectangular waveguide tunable backward wave oscillator for terahertz applications," IEEE Trans. Electron Devices, vol. 57, no. 6, pp. 1481-1484, Jun. 2010. DOI: 10.1109/TED.2010.2045678.

[22] H. Yin, L. Zhang, J. Xie, K. Ronald, W. He, G. Shu, J. Zhao, Y. Yin, X. Chen, Y. Alfadhl, A. Phelps, "Compact high-power millimetre wave sources driven by pseudospark-sourced electron beams," IET Microw. Antennas Propag., vol. 13, no. 11, pp. 1794-1798, Nov. 2019. DOI: 10.1049/iet-map.2018.6190.

[23] W. He, L. Zhang, D. Bowes, H. Yin, K. Ronald, A. Phelps, A. Cross, "Generation of broadband terahertz radiation using a backward wave oscillator and pseudospark-sourced electron beam," Appl. Phys. Lett, vol. 107, no. 13, Sep. 2015. DOI: 10.1063/1.4932099.

[24] K. Frank and J. Christiansen, "The fundamentals of the pseudospark and its applications," IEEE Trans. Plasma Sci., vol. 17, no. 5, pp. 748-753, Oct. 1989. DOI: $10.1109 / 27.41195$.

[25] H. Yin, A. W. Cross, W. He, A. Phelps, K. Ronald, "Pseudospark experiments: Cherenkov interaction and electron beam post-acceleration," IEEE Trans. Plasma Sci., vol. 32, no. 1, pp. 233-239, Feb. 2004. DOI: 10.1109/TPS.2004.823986.

[26] G. Shu, H. Yin, L. Zhang, J. Zhao, G. Liu, A. Phelps, A. W. Cross, W. He, "Demonstration of a planar W-band, kW-level extended interaction oscillator based on a pseudospark-sourced sheet electron beam," IEEE Electron Device Lett., vol. 39, no. 3, pp. 432-435, Mar. 2018. DOI: 10.1109/LED.2018.2794469

[27] G. Shu, L. Zhang, H. Yin, J. Zhao, A. Phelps, A. W. Cross, G. Liu, Y. Luo, Z. Qian, W. He, "Experimental demonstration of a terahertz extended interaction oscillator driven by a pseudospark-sourced sheet electron beam," Appl. Phys. Lett, vol.112, no.3, Jan. 2018. DOI: $10.1063 / 1.5011102$.

[28] J. Zhang, H. Yin, T. Zhang, Y. Alfadhl, X. Chen, A. Cross, W. He, J. Xie, "Design of $0.365-\mathrm{THz}$ Backward Wave Oscillator using Staggered Double Grating SWS and Sheet Beam," 12th UCMMT, London, UK, 2019, pp. 1-3, DOI: 10.1109/UCMMT47867.2019.9008255.

[29] J. Xie, L. Zhang, H. Yin, W. He, K. Ronald, A. Phelps, X. Chen, J. Zhang, Y. Alfadhl, X. Yuan, L. Meng, A. W. Cross "Study of a $0.35 \mathrm{THz}$ extended interaction oscillator driven by a pseudospark-sourced sheet electron beam," IEEE Trans. Electron Devices, vol. 67, no. 2, pp. 652658, Feb. 2020. DOI: 10.1109/TED.2019.2957760.

[30] J. Lai, Y. Gong, X. Xu, Y. Wei, Z. Duan, W. Wang, J. Feng, "W-Band 1$\mathrm{kW}$ Staggered Double-Vane Traveling-Wave Tube," IEEE Trans. Electron Devices, vol. 59, no. 2, pp. 496-503, Feb. 2012. DOI: 10.1109/TED.2011.2174458.

[31] J. P. Freund, M. A. Kodis, and N. R. Vanderplaats, "Self-consistent field theory of a helix traveling wave tube amplifier," IEEE Trans. Plasma Sci., vol. 20, no. 5, pp. 543-553, Oct. 1992. DOI: 10.1109/27.163592. 